\title{
An SMA Passive Ankle Foot Orthosis: Design, Modeling, and Experimental Evaluation
}

\author{
Liberty Deberg, ${ }^{1,2}$ Masood Taheri Andani, 1,3 \\ Milad Hosseinipour, ${ }^{1,3}$ and Mohammad Elahinia ${ }^{1}$ \\ ${ }^{1}$ Dynamic and Smart Systems Laboratory, The University of Toledo, Toledo, OH 43606, USA \\ ${ }^{2}$ Ecole Supérieure des Sciences et Technologies de l'Ingénieur de Nancy (ESSTIN), Nancy, Lorraine, France \\ ${ }^{3}$ Center for Vehicle Systems and Safety, Virginia Tech, Blacksburg, VA 24061, USA \\ Correspondence should be addressed to Masood Taheri Andani; masoodta@vt.edu
}

Received 8 January 2014; Revised 17 April 2014; Accepted 22 April 2014; Published 2 June 2014

Academic Editor: Yanjun Zheng

Copyright @ 2014 Liberty Deberg et al. This is an open access article distributed under the Creative Commons Attribution License, which permits unrestricted use, distribution, and reproduction in any medium, provided the original work is properly cited.

\begin{abstract}
Shape memory alloys (SMAs) provide compact and effective actuation for a variety of mechanical systems. In this work, the distinguished superelastic behavior of these materials is utilized to develop a passive ankle foot orthosis to address the drop foot disability. Design, modeling, and experimental evaluation of an SMA orthosis employed in an ankle foot orthosis (AFO) are presented in this paper. To evaluate the improvements achieved with this new device, a prototype is fabricated and motion analysis is performed on a drop foot patient. Results are presented to demonstrate the performance of the proposed orthosis.
\end{abstract}

\section{Introduction}

Drop foot is a motor deficiency due to paralysis of the anterior tibial muscle or the muscles innervated by the common peroneal nerve. This neuromuscular disorder can be caused by stroke, multiple sclerosis, diabetes, or neuromuscular injuries as a result of surgeries. A drop foot patient is unable to dorsiflex, hence drags the toe during swing. Moreover, the same patient is unable to control the foot during heel strike, which leads to foot slap. However, these patients often have a controlled normal plantarflexion. So far, different treatments have been developed to address this deficiency. Orthotics, functional electrical stimulation (FES), physical therapy, and surgery are among common treatments offered to these patients. Each method has specific advantages and disadvantages and is prescribed to a patient based on health conditions, symptoms, and requirements. Extensive discussion on conventional approaches is provided in [1].

Ankle foot orthoses (AFO) have increased in popularity over the past two decades. These orthotic devices have the two functions of providing stability and maintaining the range of motion. When used for permanent assistance, orthoses can enable the patient walk easier and more normally [2]. AFOs are externally applied and are intended to control the motion of the ankle, compensate the weakness, and correct the deformities. Conventional AFOs are passive mechanical braces that prevent the toe dragging by restricting the ankle movement. Although these light orthoses provide some short and long term biomechanical benefits, disadvantages still remain. Lehmann et al. [3] found that although a constant stiffness AFO was able to prevent the toe dragging, the device did not reduce the occurrence of foot slap. These devices also lead to disuse atrophy of the ankle flexor muscles by completely restricting the ankle motion. In order to address these concerns, researchers have developed active AFOs in which the impedance (stiffness) of the orthotic joint can be modulated throughout the gait. Blaya and Herr [2] developed an active ankle foot orthosis (AAFO) based on a series of elastic actuators. The main goal of this design is to change the orthosis impedance actively. As a result, the AFO minimizes the walking kinematic difference compared to a normal gait. This device includes a DC motor, mechanical linkages, and springs. Although lab tests showed promising results, the actuator weighs $2.6 \mathrm{Kg}$ and requires bulky batteries and electronics for operation.

Another active AFO uses a pneumatically powered lower limb exoskeleton [4]. This AFO is actuated by McKibben 
pneumatic artificial muscles. One pneumatic actuator provides the plantarflexion torque, while another one assists the dorsiflexion. A control algorithm adjusts the air pressure in each actuator independently. The study showed promising results in gait rehabilitation, human motor adaptation, and muscle activation. However, the applicability of these orthoses is limited to laboratory studies and rehabilitation since on board power supplies and computers are required for their operation. Shape memory alloy (SMA) based AAFOs have also been studied in recent years by several research groups $[1,5,6]$.

This paper presents the design, modeling, and evaluation of a passive AFO which utilizes the superelastic behavior of SMA wires. SMAs are a group of smart materials that can undergo large deformations and provide actuation by restoring their memorized shape. The reversible mechanism behind shape memory alloy actuation is a solid-state phase transformation that takes place in response to variation of temperature and stress. The distinct thermomechanical behavior of SMAs is the result of a transformation from the austenite (parent) phase to martensite (product) phase and vice versa [7]. These alloys have higher energy density compared to other smart materials such as piezoceramics and electroactive polymers. Therefore, actuators that implement these alloys are compact and lightweight alternatives for other conventional actuators such as DC motors and solenoids. Biocompatibility and elastic properties similar to body tissues are among the other reasons why SMAs are promising candidates for biomechanical applications and rehabilitation devices. In the following sections, the proposed design is first presented. A modeling approach is then introduced to capture the behavior of SMA wires under the required loading conditions. The proposed modeling approach is verified experimentally for a similar loading path as needed in practice. Finally, the fabricated device is experimentally tested to record the improvements in walking quality of a real drop foot subject.

\section{SMA Passive AFO Design}

A typical passive AFO is a brace with constant torsional stiffness that limits the motion of the ankle, as shown in Figure 1. The SMA passive AFO consists of a hinged brace and a series of SMA wires. This concept is depicted in Figures 2 and 3. As mentioned above, most drop foot patients are able to plantarflex their foot normally. Therefore, the superelastic wires can store mechanical energy during plantarflexion and release it during dorsiflexion to compensate the inability. Since the SMA wire undergoes stress induced phase transformation during loading and unloading, its mechanical stiffness changes. It is further shown that the change in the stiffness of the SMA wire is similar to the natural change in stiffness of the ankle joint in normal gait. This eliminates excessive loads on active muscles, letting the patient walk more naturally and feel less exhausted. On the other hand, this actuation mechanism does not require a

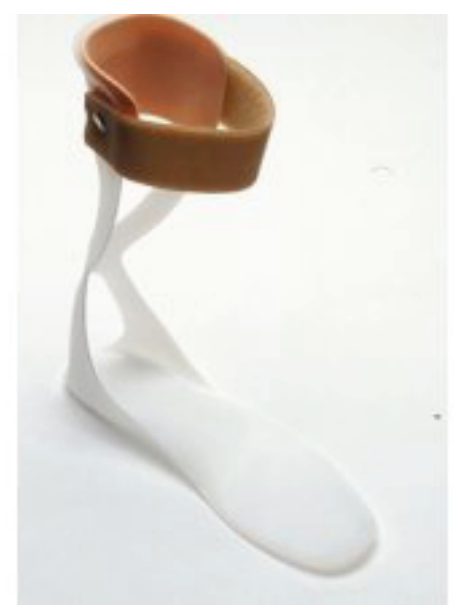

FIgURE 1: A conventional passive ankle foot orthosis (DJ Orthopedics).

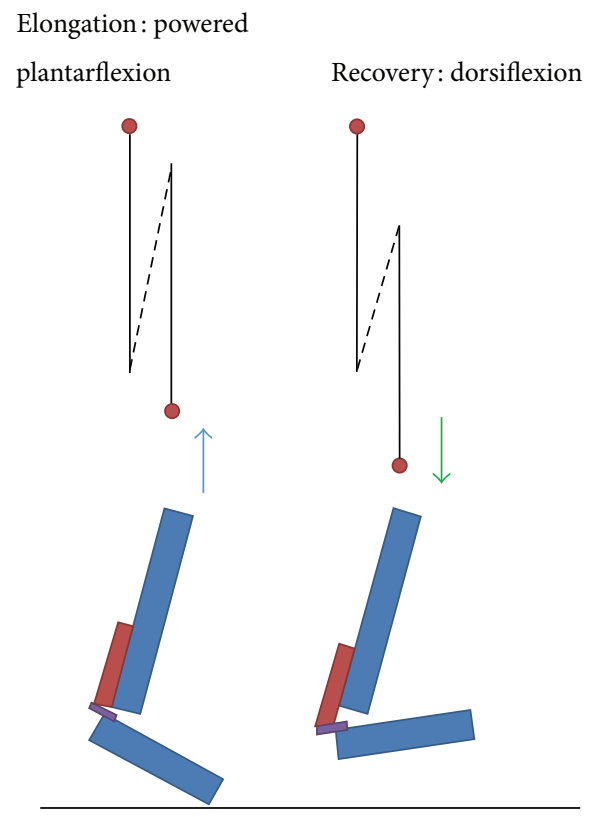

Figure 2: The SMA passive AFO concept. SMA wires store energy in plantarflexion and release it to assist during dorsiflexion.

complex controlling mechanism. Moreover, using SMA wires along with a hinged brace reduces the number of mechanical parts, manufacturing cost, and complexity of maintenance.

A prototype is developed as shown in Figure 4. The main element of the device is an SMA superelastic wire that is fixed to the brace at one end and is connected to the carriage (Figure 4) at the other end. The carriage provides sufficient freedom by moving on a slider connected to a ball joint. Several small pulleys are mounted on the brace to hold the required length of wire. The length of the wire not only affects the range of motion, but also determines the life time of the orthosis. A longer wire undergoes less strain and therefore has a longer fatigue life. 


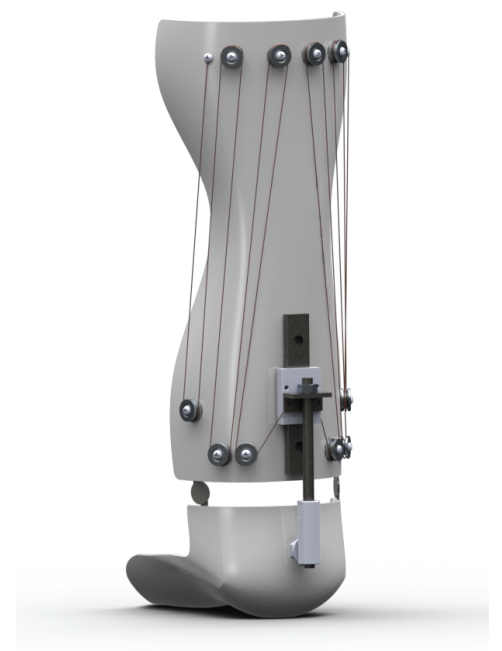

FIgure 3: The SMA passive AFO CAD design.

\section{Kinematics and Dynamics}

During a normal gait, the average walking speed is $5 \mathrm{Km} / \mathrm{h}$ and the average number of steps during one minute is about 100 [8]. Hence, one stride corresponds to $1.6 \mathrm{~m}$ during $1.2 \mathrm{~s}$. The time requirement for the AFO to provide a normal gait is thus $1.2 \mathrm{~s}$. It is notable here that different gait parameters are reported in some older classical works [9-11].

Considering that the swing phase takes about $40 \%$ of a stride, $0.48 \mathrm{~s}$ is the time to activate the wire. Foot dorsiflexes at the beginning of the swing phase to avoid the toe dragging, followed by plantarflexion prior to the heel strike. This can be seen on the ankle angle diagram in Figure 5. Angle increment corresponds to dorsiflexion and decrement corresponds to plantarflexion.

As the SMA wire embedded in the device is being extended, it provides the required rotation and torque to move the foot. In order to choose the appropriate wire, it is necessary to calculate the profile of force applied to the wire during a gait cycle. Calculations in this work are based on the tests and measurements done on a male patient in his eighties, who is $1.96 \mathrm{~m}$ tall and is $94.3 \mathrm{Kg}$ in weight [1]. If the ankle moment $(M)$ is known then the force $(F)$ applied to the wire can be found through

$$
M=F a,
$$

where $a$ is the distance between the rear of the heel and the axis of rotation of the ankle. For the subject of this study, this distance is measured to be $85 \mathrm{~mm}$. Therefore, by knowing the ankle moment, the required force can be calculated. However, the moment has not been recorded during the swing phase (and it is shown as zero in Figure 5), while the value of rotation has been recorded. Considering the weight of the foot and its acceleration (from the ankle angle diagram), the moment can be calculated during the swing phase from

$$
M=J \frac{d^{2} \theta}{d t^{2}}+m g d \cos \theta,
$$

where $J, \theta, m$, and $d$ represent the second moment of inertia, angle of rotation, mass of the foot, and the distance between the axis of rotation of ankle $(O)$ and center of gravity of the foot $(G)$. Anthropometric data are used to calculate these parameters as follows: $d=110 \mathrm{~mm}, m=1.36 \mathrm{Kg}$, and $J=0.0247 \mathrm{Kgm}^{2}[12]$.

The ankle angle data reported in Figure 5 is used to calculate the ankle moment during the swing phase from (2). Then the force profile can be found through (1). As shown in Figure 6, the maximum force needed to lift the foot up and provide the angular acceleration is $55 \mathrm{~N}$.

The next step in designing the AFO is determining the length and configuration of SMA wires. For such a purpose, a semianalytical approach is developed to capture the thermomechanical behavior of the SMA wire under the subjected loading condition.

\section{Modeling}

4.1. Coupled Constitutive Relations for the Uniaxial Loading Case. The modeling approach presented in this work is based on the 3D phenomenological constitutive model for SMAs developed by Boyd and Lagoudas [13] and Qidwai and Lagoudas [14]. The coupled form of the model is analytically studied in several works [15-17]. In this section, a reduced simple formulation of the model is presented for the situation when a wire is under uniaxial loading and unloading which is the case in the proposed SMA AFO. In this study, normal or axial strain will be considered constant throughout the crosssection, which is a valid assumption for small diameters. In this case, the stress, strain, and reversal transformation strain tensors have the following forms:

$$
\begin{gathered}
\sigma=\left(\begin{array}{ccc}
0 & 0 & 0 \\
0 & 0 & 0 \\
0 & 0 & \sigma_{z z}
\end{array}\right), \quad \epsilon=\left(\begin{array}{ccc}
\epsilon_{r r} & 0 & 0 \\
0 & \epsilon_{\theta \theta} & 0 \\
0 & 0 & \epsilon_{z z}
\end{array}\right), \\
\epsilon^{t-r}=\left(\begin{array}{ccc}
\epsilon_{r r}^{t-r} & 0 & 0 \\
0 & \epsilon_{\theta \theta}^{t-r} & 0 \\
0 & 0 & \epsilon_{z z}^{t-r}
\end{array}\right),
\end{gathered}
$$

where $\sigma_{z z}, \epsilon_{z z}$, and $\epsilon_{z z}^{t-r}$ are the axial stress, axial strain, and the axial strain at the reversal point, respectively. The terms $\epsilon_{r r}, \epsilon_{\theta \theta}, \epsilon_{r r}^{t-r}$, and $\epsilon_{\theta \theta}^{t-r}$ represent the induced normal strains and their corresponding reversal transformation strains in the two mutual perpendicular directions due to the applied axial stress $\left(\sigma_{z z}\right)$ and are introduced to meet the conservation of mass. The deviatoric stress tensor could be written as

$$
\sigma^{\prime}=\left(\begin{array}{ccc}
-\frac{\sigma_{z z}}{3} & 0 & 0 \\
0 & -\frac{\sigma_{z z}}{3} & 0 \\
0 & 0 & \frac{2 \sigma_{z z}}{3}
\end{array}\right)
$$




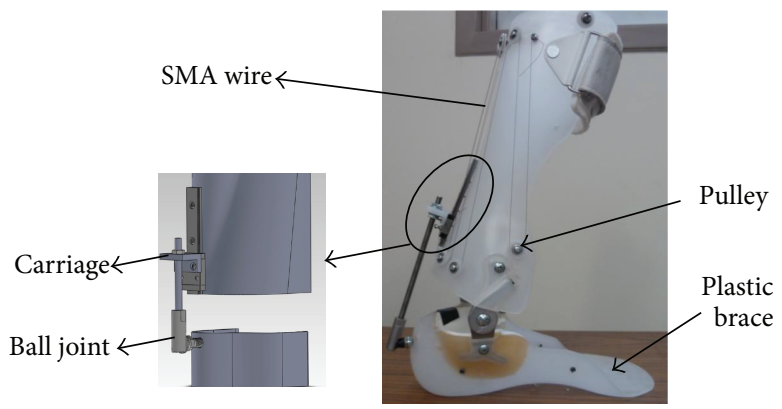

Figure 4: The SMA passive AFO prototype.

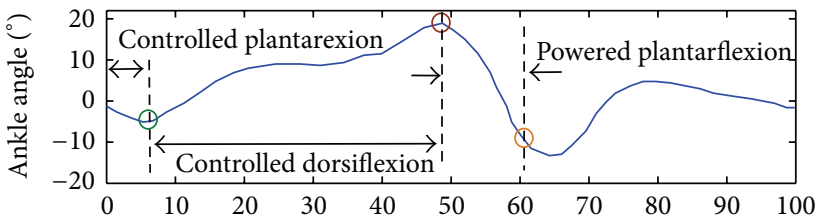

(a)

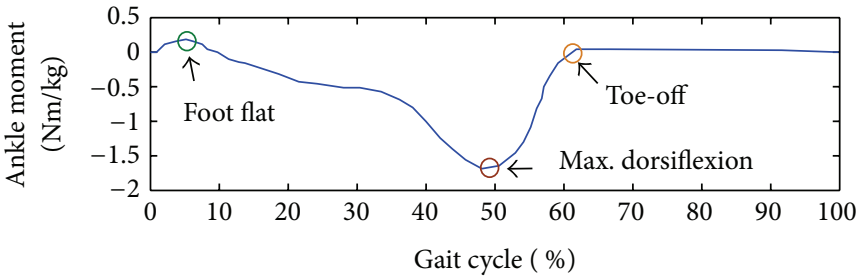

(b)

FIGURE 5: Plots of ankle angle and ankle moment versus gait percentage for a normal gait [1].

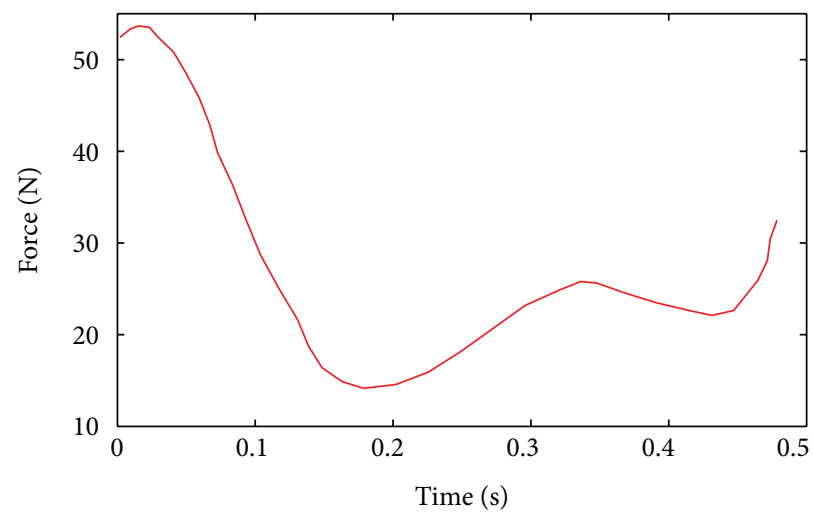

FIgURE 6: Calculated force needed during the swing phase for a drop foot patient enrolled in this study.

The transformation tensor is expressed as

$$
\begin{gathered}
\Lambda_{\mathrm{fwd}}^{t}=\frac{H}{2}\left(\begin{array}{ccc}
-1 & 0 & 0 \\
0 & -1 & 0 \\
0 & 0 & 2
\end{array}\right), \\
\Lambda_{\mathrm{rev}}^{t}=\frac{1}{\xi^{t-r}}\left(\begin{array}{ccc}
\epsilon_{r r}^{t-r} & 0 & 0 \\
0 & \epsilon_{\theta \theta}^{t-r} & 0 \\
0 & 0 & \epsilon_{z z}^{t-r}
\end{array}\right) .
\end{gathered}
$$

In (5), $\Lambda_{\text {fwd }}^{t}$ is the transformation direction tensor during forward transformation and $\Lambda_{\text {rev }}^{t}$ is that form during the reverse. It is well established that any change in the state of the system is only possible by a change in the martensitic volume fraction $\xi$. Given this assumption, the evolution of the transformation strain tensor has the following form (flow rule) $[13,14]$ :

$$
\dot{\epsilon}=\Lambda^{t} \dot{\xi}
$$

Substitute (5) into the relations of thermodynamic force and transformation function and use the following relation between the constitutive model parameters [18]:

$$
\begin{gathered}
\rho \Delta u_{0}+\mu_{1}=\frac{1}{2} \rho \Delta s_{0}\left(M_{s}+A_{f}\right), \\
Y=-\frac{1}{2} \rho \Delta s_{0}\left(A_{f}-M_{s}\right)-\mu_{2}, \\
\mu_{2}=\frac{1}{4}\left(\rho b^{A}-\rho b^{M}\right), \\
\rho b^{A}=-\rho \Delta s_{0}\left(A_{f}-A_{s}\right), \\
\rho b^{M}=-\rho \Delta s_{0}\left(M_{s}-M_{f}\right) .
\end{gathered}
$$


Then explicit expressions for the martensitic volume fraction in forward and reverse phase transformation are obtained as

$$
\begin{gathered}
\xi^{\mathrm{fwd}}=\frac{1}{\rho b^{M}}\left[\sigma_{z z} H+\frac{1}{2} \sigma_{z z}^{2} \Delta S_{33}+f^{\mathrm{fwd}}(T)\right], \\
\xi^{\mathrm{rev}}=\frac{1}{\rho b^{A}}\left[\frac{1}{\xi^{t-r}}\left(\sigma_{z z} \epsilon_{z z}^{t-r}\right)+\frac{1}{2} \sigma_{z z}^{2} \Delta S_{33}+f^{\mathrm{fwd}}(T)\right],
\end{gathered}
$$

where

$$
\begin{aligned}
& f^{\mathrm{fwd}}(T)=\rho \Delta c\left[\left(T-T_{0}\right)-T \ln \left(\frac{T}{T_{0}}\right)\right]+\rho \Delta s_{0}\left(T-M_{s}\right), \\
& f^{\mathrm{rev}}(T)=\rho \Delta c\left[\left(T-T_{0}\right)-T \ln \left(\frac{T}{T_{0}}\right)\right]+\rho \Delta s_{0}\left(T-A_{f}\right) .
\end{aligned}
$$

In (8), $\Delta S_{33}=1 / E_{M}-1 / E_{A}$, where $E$ is Young's modulus. The parameters $M_{s}$ and $A_{f}$ are the martensitic start and austenitic finish temperatures, respectively, and the superscripts fwd and rev represent forward and reverse transformations. By substituting the explicit expressions of the martensitic volume fraction equation (8) into (6) and after integrating from zero to an arbitrary time, the transformation strain can be calculated. The constitutive equations for the forward transformation are now reduced to two algebraic expressions as

$$
\begin{aligned}
\epsilon_{z z}= & \frac{1}{E_{A}+\xi^{\mathrm{fwd}}\left(E_{M}-E_{A}\right)} \sigma_{z z}+H \xi^{\mathrm{fwd}}+\alpha\left(T-T_{0}\right), \quad(10) \\
- & \frac{1}{D^{\mathrm{fwd}}}\left(-Y+\rho \Delta s_{0} T\right)\left\{\left[\frac{H}{\sigma_{z z}}+\Delta S_{33}\right] \sigma_{z z} \dot{\sigma}_{z z}\right\} \\
& +\left[\rho c-\frac{\rho \Delta s_{0}}{D^{\mathrm{fwd}}}\left(-Y+\rho \Delta s_{0} T\right)\right] \dot{T}=-\operatorname{div}(q)+\rho \widehat{g},
\end{aligned}
$$

and for the reverse transformation, the constitutive equations are

$$
\begin{gathered}
\epsilon_{z z}=\frac{1}{E_{A}+\xi^{\mathrm{rev}}\left(E_{M}-E_{A}\right)} \sigma_{z z}+\frac{\epsilon_{z z}^{t-r}}{\xi^{t-r}} \xi^{\mathrm{rev}}+\alpha\left(T-T_{0}\right) \\
-\frac{1}{D^{\mathrm{rev}}}\left(Y+\rho \Delta s_{0} T\right)\left\{\left[\frac{\epsilon_{z z}^{t-r}}{\xi^{t-r}}+\Delta S_{33} \sigma_{z z}\right] \dot{\sigma}_{z z}\right\} \\
+\left[\rho c-\frac{\rho \Delta s_{0}}{D^{\mathrm{rev}}}\left(Y+\rho \Delta s_{0} T\right)\right] \dot{T}=-\operatorname{div}(q)+\rho \widehat{g},
\end{gathered}
$$

where $v$ is Poisson's ratio that is assumed to be the same for both phases. If there is no internal heat generation source like Joule heating, then $\widehat{g}=0$. Also due to Fourier's law of thermal conduction in a cylindrical element, we can take $\operatorname{div}(q)=-k\left(\partial^{2} T / \partial r^{2}+(1 / r)(\partial T / \partial r)\right)$, where $r$ is the radius of the annular element in which the constitutive equations are studied. As shown in (11) and (13), both temperature and stress are functions of time and radius. Thus, it is necessary to define initial and boundary conditions for the problem. Initial temperature and stress distributions are prescribed as

$$
T(r, 0)=T_{\infty}, \quad \sigma_{z z}(r, 0)=0,
$$

where $T_{\infty}$ is the ambient temperature. Due to the convection at the surface of the wire and axisymmetric distribution of the temperature in the cross-section, the boundary conditions at the surface and the center of the rod can be defined as [15]

$$
\left.k \frac{\partial T(r, t)}{\partial r}\right|_{r=R_{o}}=h\left[T_{\infty}-T\left(R_{o}, t\right)\right],\left.\quad k \frac{\partial T(r, t)}{\partial r}\right|_{r=0}=0
$$

where $h$ is the heat convection coefficient and $R_{o}$ is the rod radius.

Constitutive (10) and (11) for the forward transformation and (12) and (13) for the reverse transformation, along with the initial and boundary conditions of (14) and (15), must be solved simultaneously when transformation is occurring in the material. Solving such equations is computationally very expensive using implicit schemes. Instead, an iterative approach based on the finite difference method is implemented as a MATLAB code for solving the nonlinear governing equations. The developed code takes the material properties of the SMA wire and the applied displacement profile as input and calculates the force and temperature profiles as output. Interested readers are referred to $[16,19]$ for further information on the solution procedure.

\section{Experiments}

5.1. Validation of the Coupled Model. Experiments are carried out on superelastic nitinol samples to validate the capability of the model in capturing the coupled thermomechanical behavior of an SMA wire undergoing the proposed AFO actuation pattern. An electromechanical testing machine (Bose ElectroForce 3330) equipped with temperature-controlled environmental chamber (Applied Test Systems) was used for the mechanical tests. An infrared camera (Micro-Epsilon optoNCDT 1700) is used to measure the surface temperature of the wire. The apparatus is shown in Figures 7(a) and 7(b). Two superelastic nitinol wires (NiTi number 1 provided by Fort Wayne Metals, IN) with diameters of $1.247 \mathrm{~mm}$ (0.0491 in) and $0.254 \mathrm{~mm}(0.01 \mathrm{in})$ and with gauge lengths of $27 \mathrm{~mm}$ and $100 \mathrm{~mm}$ are tested. They are referred to as wire 1 and wire 2 throughout the rest of the paper for convenience. Wires were previously annealed and surfaced with light oxide.

In order to implement the model, it is first necessary to calibrate the model with the required material properties. As a macromechanical based model, all of the required parameters can be calibrated through mechanical testing at several temperatures. In order to stabilize the stress-strain behavior of the SMA wire, a series of 30 load/unload cycles are conducted [18]. Moreover, mechanical training has to be done at a very low frequency to simulate an isothermal behavior (to avoid the latent heating effects). For such a purpose, training was conducted at $60^{\circ} \mathrm{C}$ inside the thermal chamber with the strain rate of $\dot{\epsilon}=3.7 \times 10^{-4} \mathrm{~s}^{-1}$ and axial strain 


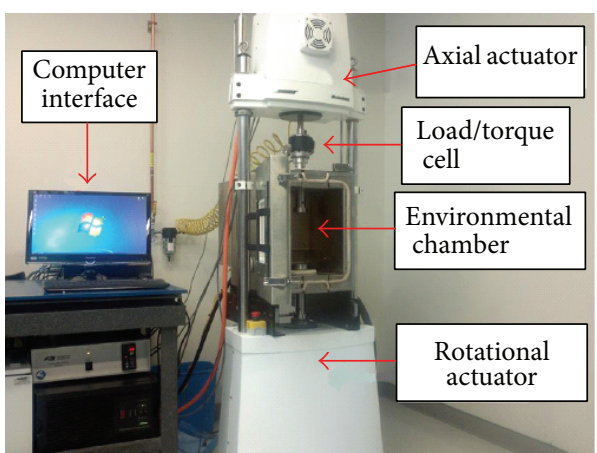

(a) Bose machine and environmental chamber

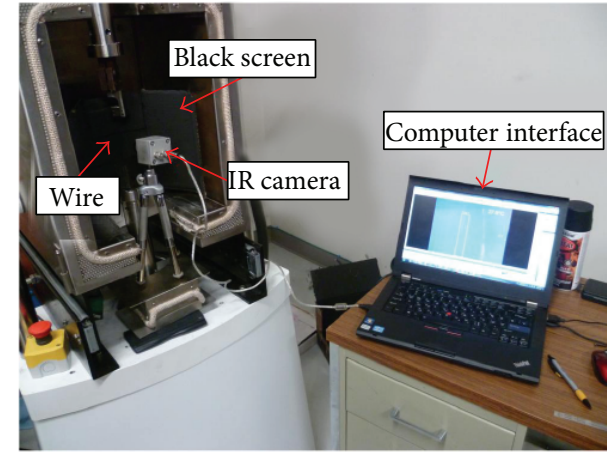

(b) Temperature measurement setup

FIGURE 7: Test setup for the model verification experiments during the experiments the environmental chamber is closed with an optical window for the IR camera.

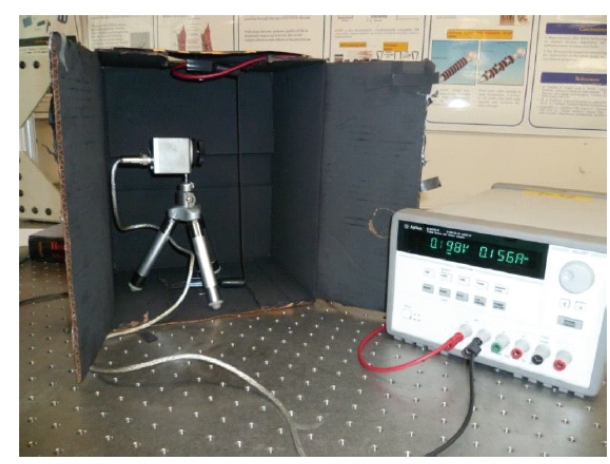

Figure 8: Apparatus of the free heat convection test. of $\epsilon=0.058$. Uniaxial tests are then performed at $60^{\circ} \mathrm{C}$, $50^{\circ} \mathrm{C}, 40^{\circ} \mathrm{C}$, and a room temperature of $23^{\circ} \mathrm{C}$ and the recorded force-displacement diagrams are used to calibrate the model parameters.

Once the wires were trained, the AFO displacement pattern is simulated. A sequence of ramps and square waves corresponding to the real path and proportional to the length of the wire is used to reproduce the strain, which wires undergo during the motion. The total time period of each cycle is set to $12 \mathrm{~s}$, which results in a frequency of $0.083 \mathrm{~Hz}$. The mechanical response (force-displacement) and the temperature variation of the wires are recorded. They are discussed and compared with the coupled model in the next sections.

5.2. Free Heat Convection of Vertical SMA Wires. Experiments are carried out to find the best empirical equation which relates the free heat convection coefficient, $h$, as a function of temperature and geometry of the wire. A superelastic nitinol wire with a diameter of $0.457 \mathrm{~mm}$ and a length of $240 \mathrm{~mm}$ is used for the test. The wire is mounted vertically inside a box and hooked up to a power supply. As seen in Figure 8, the sample and box are painted in black to attenuate the effect of ambient irradiation. The wire is heated up to an elevated temperature (e.g., $70^{\circ} \mathrm{C}$ ) by applying
TABLE 1: Calibrated material properties through mechanical testing for wire 1 and wire 2 .

\begin{tabular}{lccc}
\hline Parameter & Wire 1 & Wire 2 & Unit \\
\hline$E_{A}$ & $50.0 \times 10^{3}$ & $37.0 \times 10^{3}$ & $\mathrm{MPa}$ \\
$E_{M}$ & $27.0 \times 10^{3}$ & $27.0 \times 10^{3}$ & $\mathrm{MPa}$ \\
$M_{f}$ & 223.0 & 236.0 & $\mathrm{~K}$ \\
$M_{s}$ & 238.0 & 239.0 & $\mathrm{~K}$ \\
$A_{s}$ & 268.0 & 268.0 & $\mathrm{~K}$ \\
$A_{f}$ & 275.0 & 273.0 & $\mathrm{~K}$ \\
$C_{M}$ & 6.5 & 7.3 & $\mathrm{Mpa} / \mathrm{K}$ \\
$C_{A}$ & 7.2 & 6.8 & $\mathrm{Mpa} / \mathrm{K}$ \\
$v$ & 0.33 & 0.33 & - \\
$H$ & 0.029 & 0.038 & - \\
\hline
\end{tabular}

a constant current. After the temperature is achieved, the power supply is turned off, and the wire is left to cool down to room temperature. The associated temperature-time diagrams are recorded.

\section{Results and Discussions}

6.1. Coupled Model. The force-displacement responses of the wires at four different temperatures are used to calibrate the required material properties (the method of calibration is illustrated in [18]). These parameters are listed in Table 1. Although both specimens are NiTi number 1, they do not have exactly the same material properties due to variation in material batches and processing. However, as expected, variations are small. In addition to the properties displayed in Table 1, the model needs two more thermal parameters. These terms and their values are specific heat $\rho c=2.6 \times 10^{-6} \mathrm{~J} / \mathrm{m}^{3} \mathrm{k}$ and thermal conductivity $k=18 \mathrm{~W} /(\mathrm{mK})$. These adopted values are for a general Ni50Ti50 reported in [20] recently. The model's prediction for the simple uniaxial response of wire 2 is compared with the experiment in Figure 9. The model is in good agreement with the experiment, showing the accuracy of the calibrated parameters. 


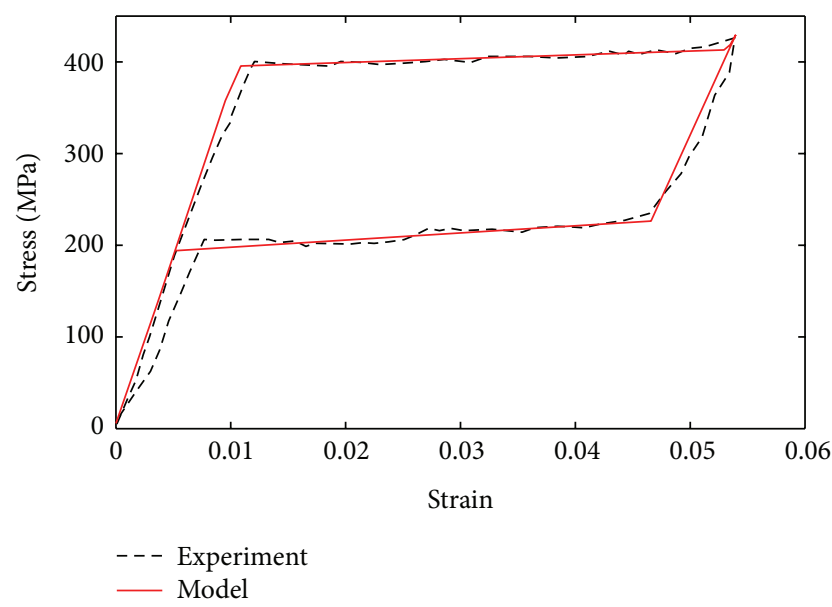

FIGURE 9: Comparison of the model's prediction with the experimental data. Uniaxial stress-strain response at room temperature for wire 2.

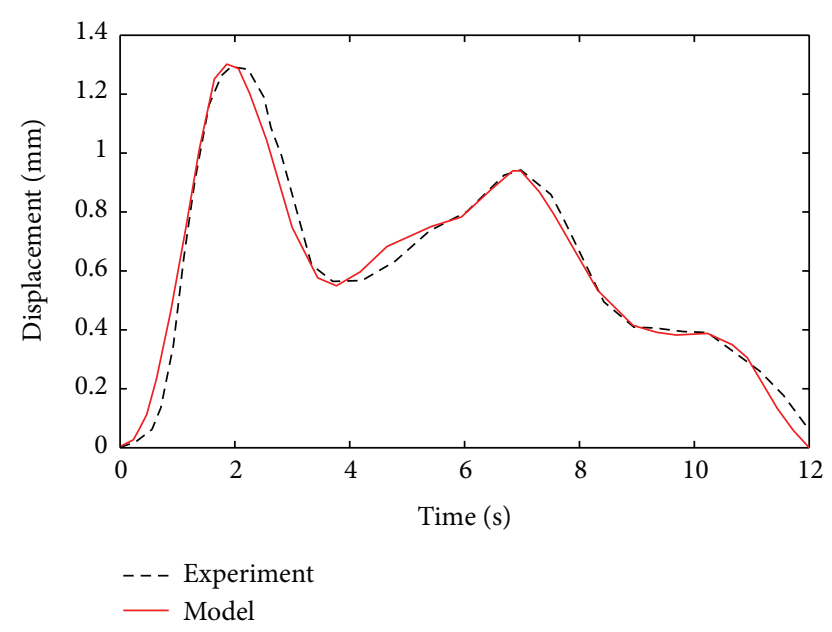

Figure 10: Comparison of the model's prediction with the experimental data. Displacement versus time diagram of wire 1 undergoing the $12 \mathrm{~s}$ AFO actuation pattern.

The recorded AFO actuation pattern during one normalized walking cycle is compared to the model's prediction in Figure 10. Although simple linear functions are used to define the loading trajectories for the mechanical testing machine, the simulated path is acceptable. Experimental stress-strain and the associated temperature-time responses of wire 1 and wire 2 are compared with the model's predictions in Figures 11(a), 11(b), 12(a), and 12(b). Results are in good agreement, showing the capability of the model in capturing the behavior of the SMA wire undergoing the AFO complex pattern.

Based on the model's prediction, it was decided to use two superelastic wires with $0.254 \mathrm{~mm}$ diameter (wire 2), in parallel configuration. The maximum force that each wire provides at the end of loading is $25 \mathrm{~N}$, which is enough for dorsiflexion of the foot.

6.2. Motion Analysis Tests. A prototype is fabricated at the Dynamic and Smart Systems Laboratory, The University of

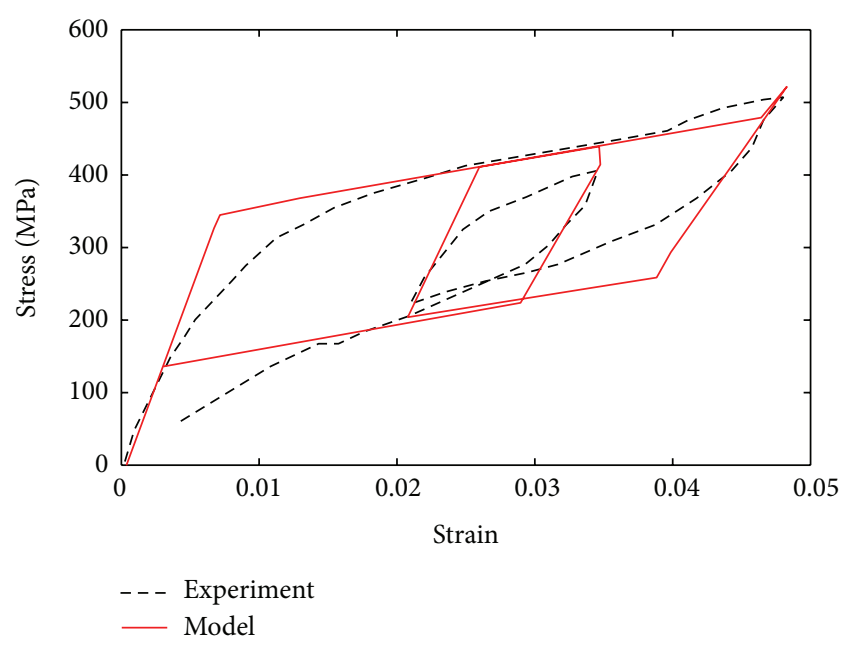

(a) Stress versus strain diagram

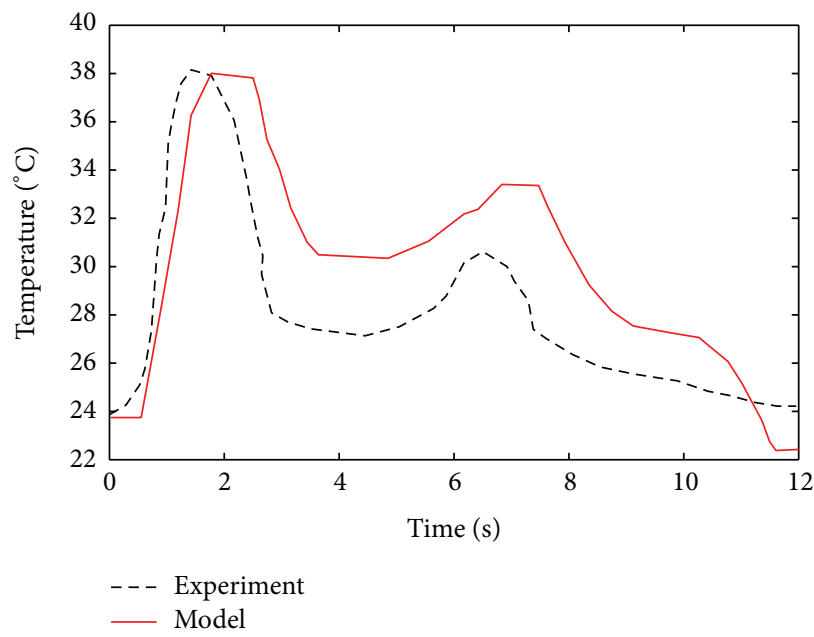

(b) Temperature versus time diagram

FIGURE 11: Comparison of the model's prediction with the experimental data of wire 1 undergoing the $12 \mathrm{~s}$ AFO actuation pattern.

Toledo. In order to evaluate the performance of the prototype, gait analyses are performed on a real subject. Data is collected to compare the ankle stiffness characteristics of a drop foot patient with and without an AFO. To demonstrate the improvements achieved with the new device, three sets of tests are performed: without AFO, with a hinged AFO, and finally with the proposed SMA AFO. This patient is diagnosed with left leg drop foot and wears a brace for his daily activities. Figure 13 shows the patient wearing the SMA AFO for the motion analysis test.

Ankle moment and angle data are collected for each test. Figure 14 compares the ankle angle versus gait percentage for the three cases. In absence of any AFO, there is a negative angle at the end of the cycle, which means that the patient is not able to raise his foot during dorsiflexion. Although wearing a typical brace helped him raise his foot and walk more naturally, there is still a large amount of residual negative angle at the end of the gait. While wearing the SMA 


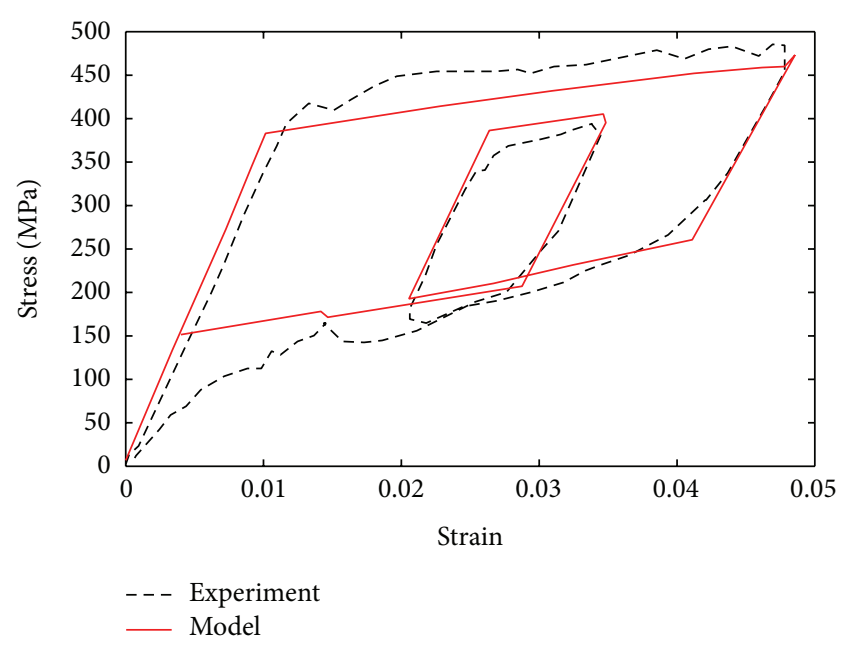

(a) Stress versus strain diagram

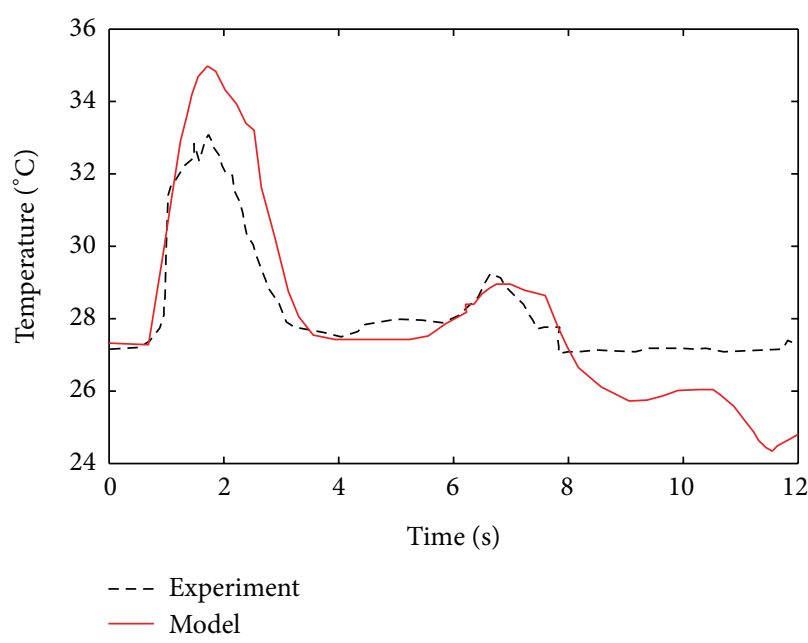

(b) Temperature versus time diagram

FIGURE 12: Comparison of the model's prediction with the experimental data of wire 2 undergoing the $12 \mathrm{~s}$ AFO actuation pattern.

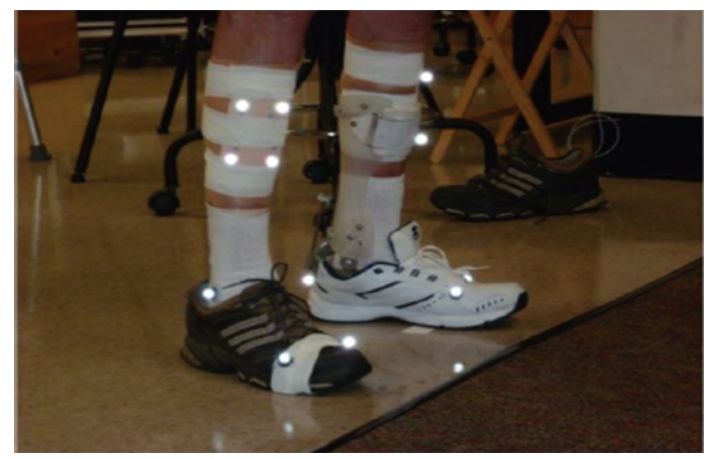

(a)

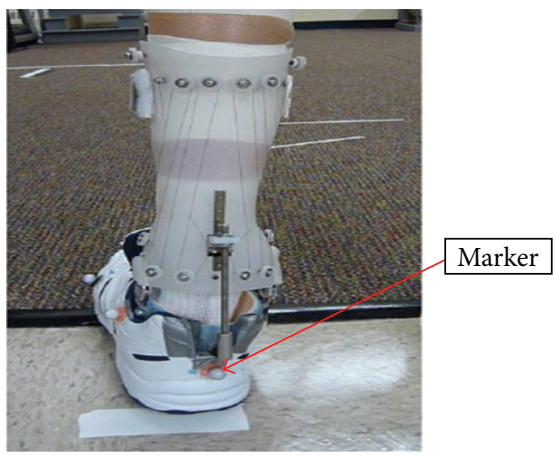

(b)

FIGURE 13: Patient wearing the SMA AFO for the motion analysis test.

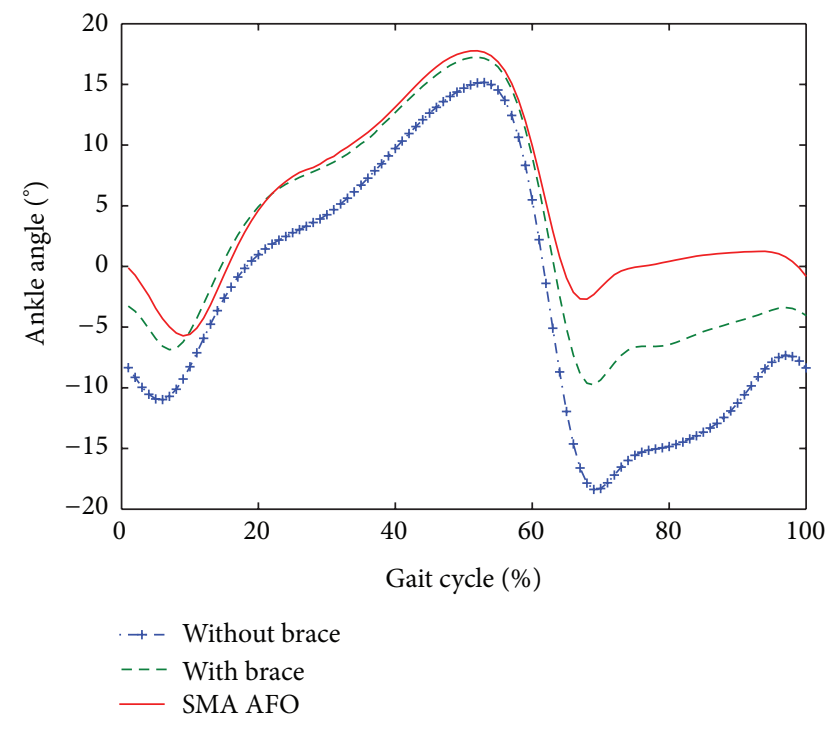

FIGURE 14: Ankle angle (mean value) versus gait percentage for three different tests.
AFO, he was able to dorsiflex his foot as desired, which is much closer to a normal gait.

Since only one of the patient's feet is diagnosed with drop foot, it is possible to compare the ankle angle and moment angle responses of the healthy foot with the other foot while wearing the proposed AFO. These comparisons are shown in Figures 15(a) and 15(b). Regardless of a small deviation from the desired pattern, the important accomplishment for the device is in giving the patient the ability to recover the moment and angle at the end of the gait. A notable point here is that due to atrophy, muscles of the foot suffering from drop foot are not as strong as those of a healthy one; thus, exactly the same angle and moment profiles could not be expected for the two feet.

\section{Conclusions}

A superelastic SMA wire was used to develop a passive ankle foot orthosis for addressing the drop foot disability. The main benefit of the new AFO compared to the other conventional ones was providing fast and effective actuation stroke with a 


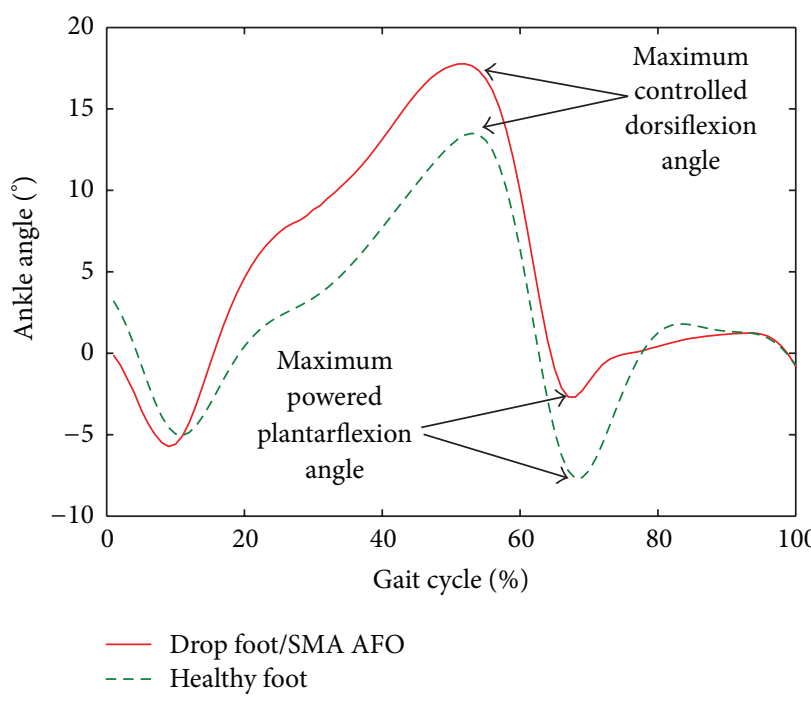

(a) Ankle angle versus gait percentage

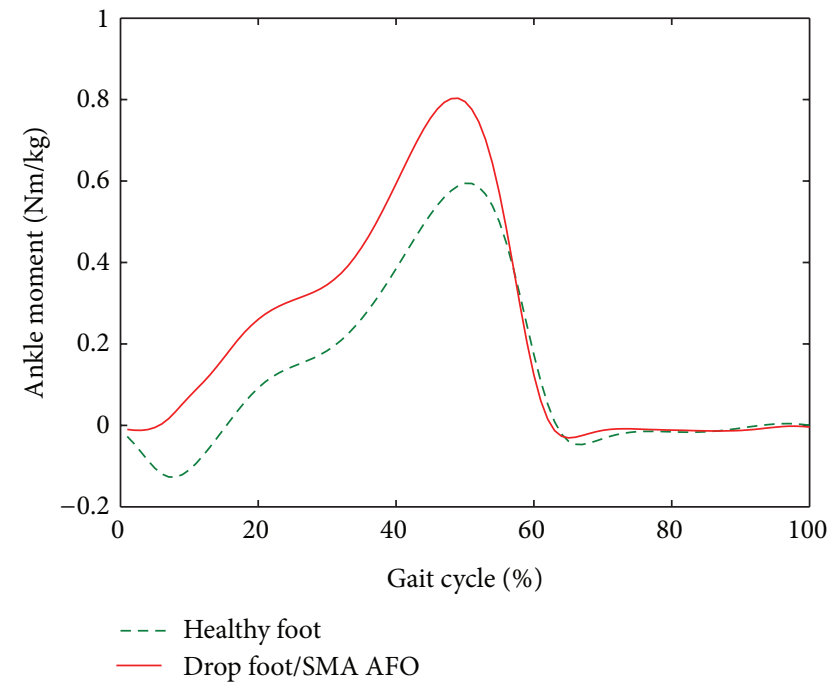

(b) Ankle moment versus gait percentage

FIGURE 15: Comparison of normal angle (a) and moment (b) profiles with SMA AFO assisted drop foot condition.

simple design. A comprehensive coupled thermomechanical model was used for the SMA that enabled design evaluation and optimization. This model included the SMA latent heat effect and free heat convection phenomenon and captured the dynamic rate dependent response of the material. A prototype of the device was designed based on the model and fabricated. Motion analysis was conducted to evaluate the effectiveness of the device. The SMA AFO presented in this work demonstrated the ability to meet the torque-angle requirements of an ankle assistive device much better than a typical brace or a conventional passive AFO.

\section{Appendix}

\section{Heat Convection}

Several sets of empirical formulas are given in the literature to obtain the free heat convection coefficient of a vertical slender cylinder. The Nusselt number for a slender cylinder is shown by Popiel et al. [21] to be found as

$$
N u=\frac{4}{3}\left[\frac{7 \operatorname{RaPr}}{5(20+21 \mathrm{Pr})}\right]^{0.25}+\frac{4 L(272+315 \mathrm{Pr})}{35 d(64+63 \mathrm{Pr})},
$$

where $d$ and $L$ are the diameter and length of the wire, respectively. $\mathrm{Ra}=\mathrm{GrPr}$ is the Rayleigh number and $\mathrm{Pr}$ is the Prandtl number of the air at ambient temperature. $\mathrm{Gr}=g \beta\left(T-T_{\infty}\right) L^{3} / \nu^{2}$ is the Grashof number, where $g$ is the gravitational acceleration; $\beta$ is the volume coefficient of expansion, that is, $\beta=1 / T$ for ideal gases; $T$ is the wall temperature; $T_{\infty}$ is the ambient temperature; and $v$ is the kinematic viscosity of air. Having the Nusselt number, the free convection coefficient is calculated by $h=(N u \cdot k) / L$, where $k$ is the air thermal conductivity of air. Popiel [22] suggested that the Nusselt number of a vertical slender cylinder can be found by calculating the Nusselt number of a flat plate and then applying a correction. He proposed the following equation for the Nusselt number of a flat plate:

$$
N u_{\mathrm{FP}}=\left[0.825+\frac{0.387 \mathrm{Ra}^{1 / 6}}{\left(1+(0.492 \mathrm{Pr})^{9 / 16}\right)^{8 / 27}}\right]^{2},
$$

and the correction he used is

$$
\frac{N u}{N u_{\mathrm{FP}}}=1+0.3\left(32^{0.5} \mathrm{Gr}^{-0.025} \frac{L}{D}\right) .
$$

Churchill and Chu [23] suggested another empirical expression to calculate the Nusselt number of a flat plate as

$$
N u_{\mathrm{FP}}=\left[0.68+\frac{0.67 \mathrm{Ra}^{0.25}}{\left(1+(0.49 \mathrm{Pr})^{0.56}\right)^{0.44}}\right],
$$

and they used the same correction as (A.3) to find the Nusselt number of a vertical slender cylinder.

In order to calculate the experimental value of $h$, one might start from the heat transfer equation. Ignoring the heat convention through the wire, the heat transfer equation for an SMA element could be written as

$$
m C_{p} \frac{\partial T}{\partial t}=P+h A\left(T-T_{\infty}\right)+m \Delta H \frac{\partial \xi}{\partial t},
$$

where $m, C_{p}, P$, and $h$ are the mass, heat capacity, dissipated power, and free convective heat coefficient, respectively. Parameters $\xi$ and $\Delta H$ represent the martensitic volume fraction and latent heat. In the experiment conducted, no transformation occurs and only the cooling period is recorded; thus, the terms $\Delta H$ and $P$ are both zero. Therefore, (A.5) can be rewritten as

$$
\rho \frac{\pi d^{2}}{4} L C_{p}=-h \pi d L\left(T-T_{\infty}\right),
$$




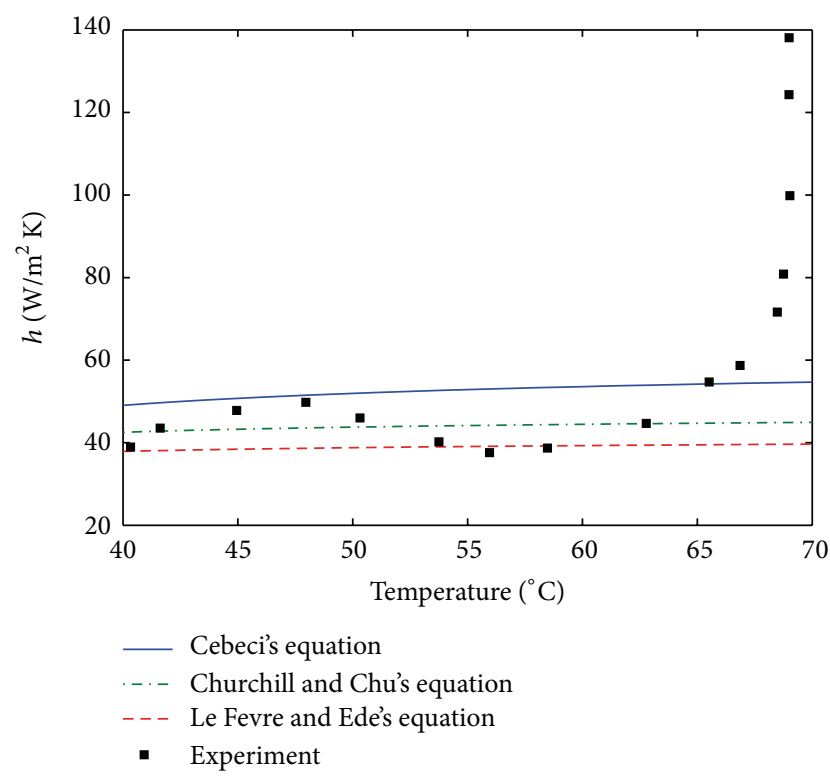

Figure 16: Comparison between the experimental data and the empirical equations available in the literature for the heat convection coefficient versus temperature.

which results in the following expression for $h$ :

$$
h=-\frac{\rho d C_{p}}{4\left(T-T_{\infty}\right)} \frac{\Delta T}{\Delta t} .
$$

In (A.6), the term $\rho$ represents the material density. Equation (A.7) is used to calculate $h$ from temperature-time response of the wire recorded during the cooling period in the experiment. The experimental values of $h$ are then compared against the predicted values by the empirical equations. As shown in Figure 16, all equations are in good agreement with the experiment. However, Cebeci's equation is chosen since it does not underestimate the results and thus is more reliable for design purposes.

\section{Conflict of Interests}

The authors declare that there is no conflict of interests regarding the publication of this paper.

\section{Acknowledgments}

The authors would like to acknowledge the financial support provided from Nitinol Commercialization Accelerator by the Ohio Department of Development through Grant WP 10-010. NSF support through award 0731087 Research to Aid Person with Disability is also appreciated. Assistance of Michael Steves from Marshall-Loene Orthopedics Inc., Maummy, $\mathrm{OH}$, in fabrication is also appreciated.

\section{References}

[1] M. Bhadane-Deshpande, Towards a shape memory alloy based variable stiffness ankle foot orthosis, [Ph.D. thesis], University of Toledo, Toledo, Ohio, USA, 2012.
[2] J. A. Blaya and H. Herr, "Adaptive control of a variableimpedance ankle-foot orthosis to assist drop-foot gait," IEEE Transactions on Neural Systems and Rehabilitation Engineering, vol. 12, no. 1, pp. 24-31, 2004.

[3] J. F. Lehmann, S. M. Condon, B. J. De Lateur, and R. Price, "Gait abnormalities in peroneal nerve paralysis and their corrections by orthoses: a biomechanical study," Archives of Physical Medicine and Rehabilitation, vol. 67, no. 6, pp. 380-386, 1986.

[4] D. P. Ferris, J. M. Czerniecki, and B. Hannaford, "An ankle-foot orthosis powered by artificial pneumatic muscles," Journal of Applied Biomechanics, vol. 21, no. 2, pp. 189-197, 2005.

[5] S. Pittaccio, S. Viscuso, M. Rossini et al., "SHADE: a shapememory-activated device promoting ankle dorsiflexion," Journal of Materials Engineering and Performance, vol. 18, no. 5-6, pp. 824-830, 2009.

[6] S. Pittaccio, S. Viscuso, E. Beretta, A. C. Turconi, and S. Strazzer, "Pilot studies suggesting new applications of NiTi in dynamic orthoses for the ankle joint," Prosthetics and Orthotics International, vol. 34, no. 3, pp. 305-318, 2010.

[7] L. C. Brinson, "One-dimensional constitutive behavior of shape memory alloys: thermomechanical derivation with nonconstant material functions and redefined martensite internal variable," Journal of Intelligent Material Systems and Structures, vol. 4, no. 2, pp. 229-242, 1993.

[8] C.-C. Yang, Y.-L. Hsu, K.-S. Shih, and J.-M. Lu, "Real-time gait cycle parameter recognition using a wearable accelerometry system," Sensors, vol. 11, no. 8, pp. 7314-7326, 2011.

[9] J. Perry and J. R. Davids, "Gait analysis: normal and pathological function," Journal of Pediatric Orthopaedics, vol. 12, no. 6, p. 815, 1992.

[10] T. Oberg, A. Karsznia, and K. Oberg, "Basic gait parameters: reference data for normal subjects, 10-79 years of age," Journal of Rehabilitation Research and Development, vol. 30, no. 2, pp. 210-223, 1993.

[11] J. R. Gage, P. A. Deluca, and T. S. Renshaw, "Gait analysis: principles and applications," Journal of Bone and Joint Surgery $A$, vol. 77, no. 10, pp. 1607-1623, 1995.

[12] L. Deberg, A fast actuator using shape memory alloys for an ankle foot orthosis, [Master's thesis], Ecole Superieure des Sciences et Technologies de l'Ingenieur de Nancy, 2012.

[13] J. G. Boyd and D. C. Lagoudas, "A thermodynamical constitutive model for shape memory materials. Part I. The monolithic shape memory alloy," International Journal of Plasticity, vol. 12, no. 6, pp. 805-842, 1996.

[14] M. A. Qidwai and D. C. Lagoudas, "Numerical implementation of a shape memory alloy thermomechanical constitutive model using return mapping algorithms," International Journal for Numerical Methods in Engineering, vol. 47, no. 6, pp. 1123-1168, 2000.

[15] R. Mirzaeifar, R. Desroches, and A. Yavari, "Analysis of the rate-dependent coupled thermo-mechanical response of shape memory alloy bars and wires in tension," Continuum Mechanics and Thermodynamics, vol. 23, no. 4, pp. 363-385, 2011.

[16] M. T. Andani, A. Alipour, and M. Elahinia, "Coupled ratedependent superelastic behavior of shape memory alloy bars induced by combined axial-torsional loading: a semi-analytic modeling," Journal of Intelligent Material Systems and Structures, vol. 24, no. 16, pp. 1995-2007, 2013.

[17] R. Mirzaeifar, R. Desroches, A. Yavari, and K. Gall, "Coupled thermo-mechanical analysis of shape memory alloy circular 
bars in pure torsion," International Journal of Non-Linear Mechanics, vol. 47, no. 3, pp. 118-128, 2012.

[18] D. Lagoudas, Shape Memory Alloys: Modeling and Engineering Applications, Springer, New York, NY, USA, 2008.

[19] M. T. Andani, A. Alipour, A. Eshghinejad, and M. Elahinia, "Modifying the torque-angle behavior of rotary shape memory alloy actuators through axial loading: a semi-analytical study of combined tension-torsion behavior," Journal of Intelligent Material Systems and Structures, vol. 24, no. 12, pp. 1524-1535, 2013.

[20] R. Mirzaeifar, R. Desroches, and A. Yavari, "Analysis of the rate-dependent coupled thermo-mechanical response of shape memory alloy bars and wires in tension," Continuum Mechanics and Thermodynamics, vol. 23, no. 4, pp. 363-385, 2011.

[21] C. O. Popiel, J. Wojtkowiak, and K. Bober, "Laminar free convective heat transfer from isothermal vertical slender cylinder," Experimental Thermal and Fluid Science, vol. 32, no. 2, pp. 607613, 2007.

[22] C. O. Popiel, "Free convection heat transfer from vertical slender cylinders: a review," Heat Transfer Engineering, vol. 29, no. 6, pp. 521-536, 2008.

[23] S. W. Churchill and H. H. S. Chu, "Correlating equations for laminar and turbulent free convection from a vertical plate," International Journal of Heat and Mass Transfer, vol. 18, no. 11, pp. 1323-1329, 1975. 

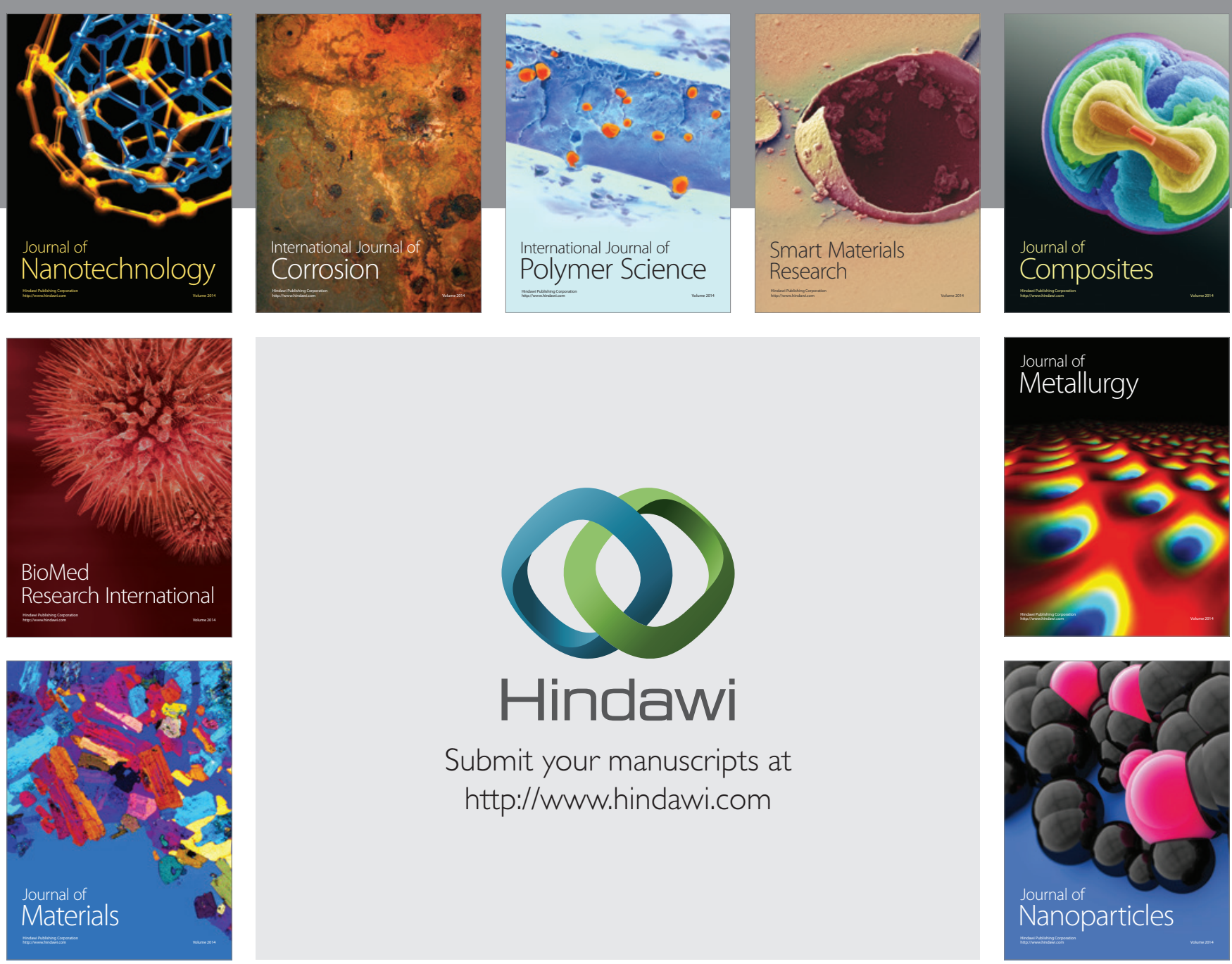

Submit your manuscripts at http://www.hindawi.com
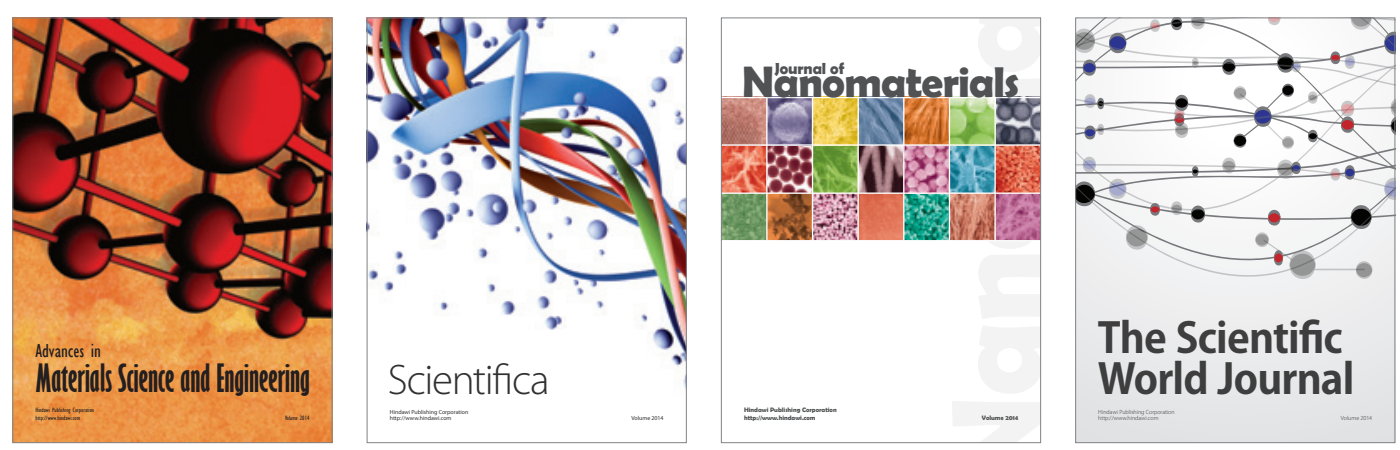

\section{The Scientific World Journal}
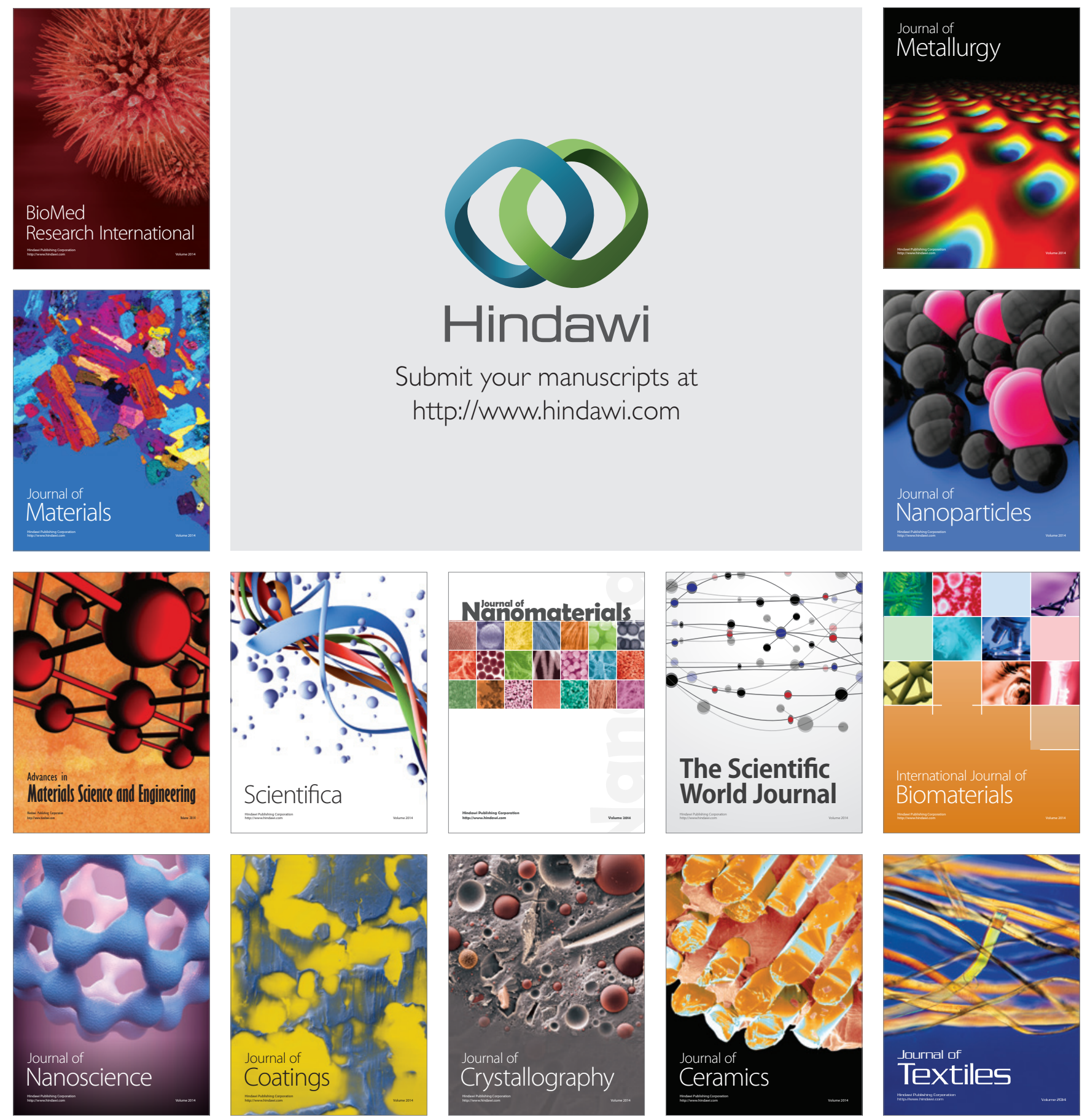